\title{
Expression of PD-1 and PD-L1 in thymic epithelial neoplasms
}

\author{
Annikka Weissferdt ${ }^{1}$, Junya Fujimoto ${ }^{2}$, Neda Kalhor ${ }^{1}$, Jaime Rodriguez ${ }^{2}$, Roland Bassett ${ }^{3}$, \\ Ignacio I Wistuba ${ }^{2}$ and Cesar A Moran ${ }^{1}$ \\ ${ }^{1}$ Department of Pathology, MD Anderson Cancer Center, Houston, TX, USA; ${ }^{2}$ Department of Translational \\ Molecular Pathology, MD Anderson Cancer Center, Houston, TX, USA and ${ }^{3}$ Department of Biostatistics, MD \\ Anderson Cancer Center, Houston, TX, USA
}

\begin{abstract}
Thymic epithelial neoplasms are rare tumors that are difficult to diagnose and treat. Programmed death 1 (PD-1) receptor and its ligand (PD-L1) are expressed by various malignancies and are considered a prognostic factor and immunotherapeutic target. We examined the expression of both antibodies in 100 thymic epithelial neoplasms to assess their use as a biomarker and to correlate their expression with clinicopathological parameters. Whole-tissue sections of 74 thymomas and 26 thymic carcinomas were examined. Expression of PD-1 and PD-L1 was evaluated by immunohistochemistry and scored by the percentage of positive T-cells or tumor cells, respectively. Cases with strong membranous reactivity of the antibody in $\geq 5 \%$ of T-cells (PD-1) or tumor cells (PD-L1), respectively, were considered positive. Expression of PD-1 was detected in 52/100 cases (52\%) including 6/26 thymic carcinomas (23\%) and 46/74 thymomas (62\%). PD-L1 was positive in $61 / 100$ cases $(61 \%)$ including $14 / 26$ thymic carcinomas (54\%) and $47 / 74$ thymomas (64\%). A total of 82 cases (82\%) showed expression of PD-1 or PD-L1. PD-1+ cases were associated with higher stage in thymic carcinoma $(P=0.01)$ and PD-1 ${ }^{-}$cases with thymic carcinoma histology $(P=0.0014)$, whereas $\mathrm{PD}-\mathrm{L} 1^{+}$cases were associated with neoadjuvant therapy in thymoma $(P=0.0065)$. There was no statistical difference between PD-1 or PD-L1 expression status and other clinicopathological parameters including overall survival. PD-1 and/or PD-L1 are expressed in up to $82 \%$ of thymic epithelial neoplasms. These results confirm that these tumors should be considered for PD-1/PD-L1-targeted therapy, however their predictive value in terms of prognosis remains uncertain.

Modern Pathology (2017) 30, 826-833; doi:10.1038/modpathol.2017.6; published online 10 March 2017
\end{abstract}

Thymic epithelial neoplasms are rare tumors with an incidence of $<1 \%$ of all adult cancers. ${ }^{1,2}$ They are divided into thymomas and thymic carcinomas. Although thymic carcinomas are overtly malignant epithelial tumors, thymomas exhibit at least low grade malignant behavior with the potential for invasion and metastasis. The rarity of these tumors coupled with their morphological heterogeneity, has to date precluded any larger clinical trials and the treatment for these tumors is inconsistent, consisting mainly of multimodal treatment including complete surgical resection and chemotherapy and/or radiotherapy. In the last two decades, adjunct techniques such as immunohistochemistry and molecular investigations have emerged with the aim to facilitate

Correspondence: Dr A Weissferdt, MD, FRCPath, Department of Pathology, MD Anderson Cancer Center, 1515 Holcombe Boulevard, Houston, TX 77030, USA.

E-mail: aweissferdt@mdanderson.org

Received 17 October 2016; revised 29 December 2016; accepted 30

December 2016; published online 10 March 2017 diagnosis and tailor medical treatment. However, so far no consistent molecular abnormality has emerged that would define thymic tumors. ${ }^{3}$ Hence, personalized treatment approaches like those already in practice for other tumors are still lacking.

An emerging new strategy in cancer treatment is the blockade of immune checkpoints. ${ }^{4}$ The development of effective cancer immunotherapies depends on reversing inhibitory stimulus in the tumor microenvironment, thereby enhancing the visibility of tumor cells to the immune system. Programmed death 1 (PD-1) is a key immune checkpoint receptor, which mediates immunosuppression and is expressed by activated T cells. PD-1 functions primarily in peripheral tissues, where $\mathrm{T}$ cells, including tumor infiltrating lymphocytes may encounter the immunosuppressive PD-1 ligand PD-L1 which is expressed by tumor cells, stromal cells, or both. Inhibition of this pathway using blocking monoclonal antibodies against PD-1 or PD-L1 is emerging as an effective method for reversing cancer immunosuppression and can lead to tumor regression in patients with 
Table 1 Antibody information

\begin{tabular}{|c|c|c|c|c|}
\hline Antibody & Clone & Vendor & Dilution & Staining pattern \\
\hline PD-1 & E1L3N & Cell Signaling Technology, Danvers, MA, USA & $1: 100$ & Membranous \\
\hline PD-L1 & EPR4877(2) & Abcam, Cambridge, MA, USA & $1: 250$ & Membranous \\
\hline
\end{tabular}

Abbreviations: PD-1, programmed death 1; PD-L1, programmed death ligand 1.

advanced disease. ${ }^{4-6}$ Immunohistochemical studies for PD-1 and its ligand performed on tumor tissue can identify patients who are likely to respond to antiPD-1/PD-L1 therapy. As such, response to this type of treatment has been shown for a number of different cancer types including non-small cell lung carcinoma, central nervous system tumors, melanoma, and lymphoma among others. ${ }^{7-11}$ To investigate the use of PD-1 and PD-L1 as potential biomarkers for thymic epithelial neoplasms, we examined the immunohistochemical expression of these markers in a series of 100 cases comprising thymomas and thymic carcinomas and correlated the expression status with clinicopathological parameters.

\section{Materials and methods}

One hundred cases of thymic epithelial neoplasms were obtained from the surgical pathology files of MD Anderson Cancer Center, Houston. Whole-tissue sections from surgical resection specimens were available in all cases. Pathologic diagnosis and tumor classification was performed according to the system proposed by Suster and Moran. ${ }^{12}$ A near equal number of cases from each tumor category including 25 spindle cell thymomas (WHO type A), 25 conventional thymomas (WHO type B1 and B2), 24 atypical thymomas (WHO B3), and 26 thymic carcinomas (18 squamous cell carcinomas, 5 highgrade carcinomas, not otherwise specified, and 3 basaloid carcinomas) were selected for inclusion in this study. All tumors were pure subtypes; tumors with mixed histology were excluded from analysis. Tumor staging was performed according to the systems proposed by Moran et $a l^{13}$ for thymomas and Weissferdt and Moran ${ }^{14}$ for thymic carcinomas. Clinical data and follow-up were obtained from the patients' medical charts.

Immunohistochemical studies were performed on formalin-fixed paraffin-embedded whole-tissue sections from each tumor. The sections were incubated with $3 \% \mathrm{H}_{2} \mathrm{O}_{2}$ in methanol and fetal bovine serum to block endogenous peroxidase activity and nonspecific protein-protein interactions, respectively. Immunostaining was performed using a horseradish peroxidase-labeled polymer system. Tissue sections were incubated with antibodies against PD-1 and PD-L1 (Table 1). Diaminobenzidine was used as a chromogen for antigen localization. Adequate positive and negative controls were run for both antibodies. The percentage of positive tumor infiltrating lymphocytes (PD-1) or tumor cells (PD-L1) was evaluated and scored. Cases with strong membranous reactivity of the antibody on $\geq 5 \%$ of T-cells or tumor cells, respectively, were considered positive. The cut-off of $5 \%$ was chosen based on previous publications. . $^{5,7,9}$

The method of Kaplan and Meier was used to estimate the distribution of overall survival from the date of surgery. Distributions were compared using the log-rank test. Fisher's exact test was used to compare the distribution of variables [tumor stage, histologic type, lymph nodes status (for thymic carcinoma], and neoadjuvant therapy) between PD-1 and PD-L1 positive and negative patients, respectively. All statistical analyses were performed using $\mathrm{R}$ version 3.2.2. All statistical tests used a significance level of $5 \%$. Analyses were performed separately in thymic carcinoma and thymoma patients because of the known difference in biologic behavior and prognosis.

\section{Results}

\section{Clinicopathologic Characteristics}

The patients were 55 males and 42 females with an average age of 55 years (range, 19-84 years). Twentysix cases were classified as thymic carcinoma (Figure 1a) including 18 squamous cell carcinomas, 5 high-grade carcinomas, not otherwise specified, and 3 basaloid carcinomas, and 72 cases were classified as thymoma including 25 spindle cell thymomas (WHO type A) (Figure 1b), 25 conventional thymomas (WHO types B1 and B2) (Figure 1C), and 24 atypical thymomas (WHO type B3) (Figure 1d). Nineteen of the thymoma patients $(26 \%)$ had an associated autoimmune disorder; no such association was noted for any of the thymic carcinoma patients. Among the thymoma patients, 8 presented with Moran stage 0 (11\%), 33 with stage 1 (46\%), 10 with stage 2a (14\%), 5 with stage $2 \mathrm{~b}(7 \%), 1$ with stage 2c (1\%), 14 with stage $3 \mathrm{a}(20 \%)$, and 1 with stage $3 \mathrm{~b}$ tumors $(1 \%)$. The thymic carcinoma patients were staged as follows: 8 stage I (31\%), 11 stage II ( $42 \%)$, and 6 stage III (23\%) according to Weissferdt and Moran. ${ }^{14}$

\section{Immunohistochemical Results}

Expression of PD-1 was restricted to tumor infiltrating lymphocytes and detected in 6/26 thymic carcinomas (23\%, Figure 1e), 15/25 spindle cell thymomas (WHO type A) (60\%, Figure 1f), 19/25 

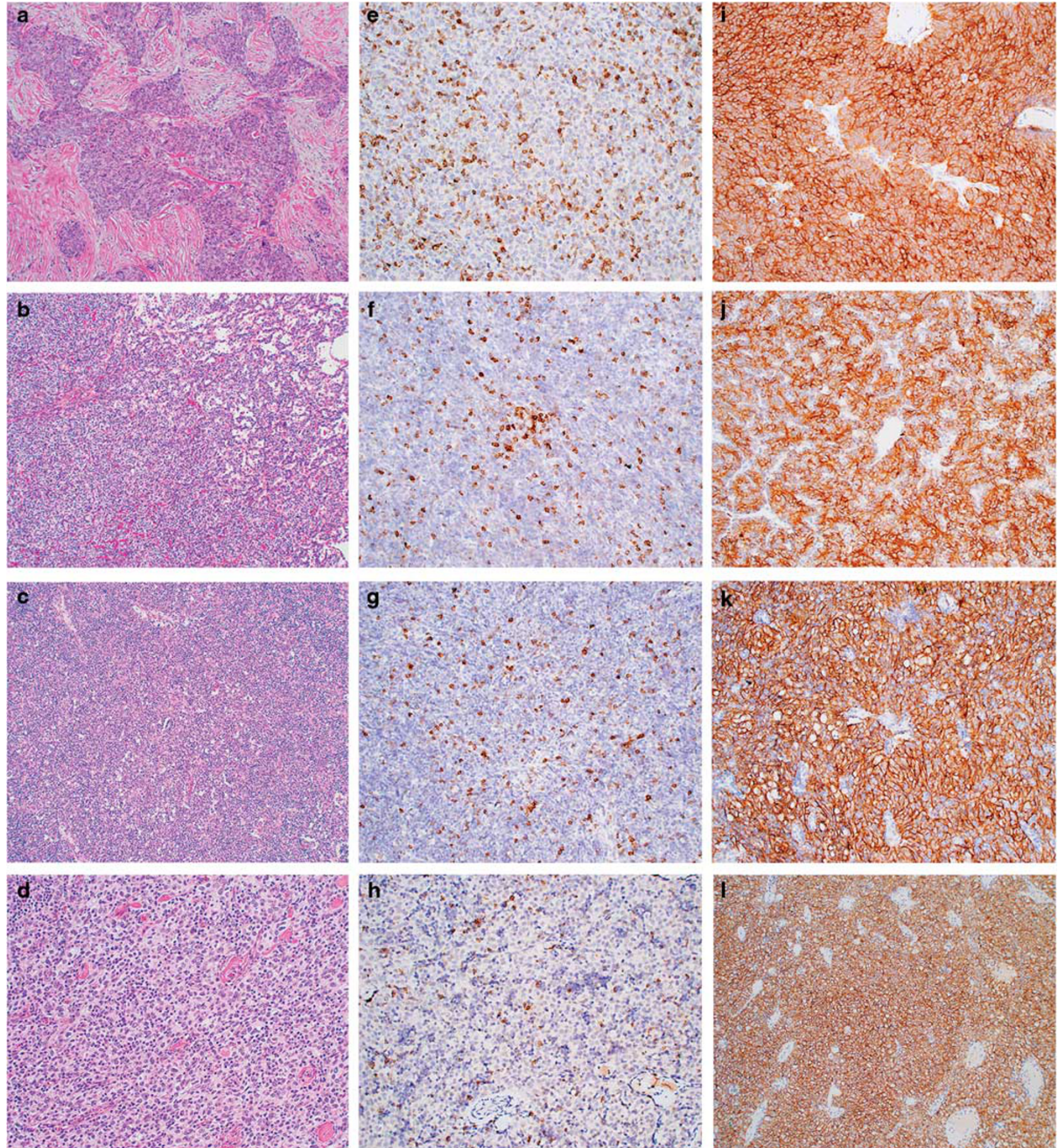

Figure 1 (a) Thymic carcinoma characterized by islands of tumor cells with malignant cytologic features embedded in dense desmoplastic stroma (H\&E). (b) Spindle cell thymoma with diffuse (lower left) and adenomatoid (upper right) growth patterns (H\&E). (c) Conventional thymoma composed of epithelial cells and abundant lymphocytes (H\&E). (d) Atypical thymoma showing a prominent epithelial component and only sparse lymphocytes. Mild cytologic atypia can be noted (H\&E). (e) PD-1 positive lymphocytes infiltrate the tumor cells in a thymic carcinoma. (f) A case of PD-1 positive tumor infiltrating lymphocytes among the spindle cell thymoma group. (g) The majority of conventional thymomas were PD-1 positive. (h) Atypical thymoma with PD-1 positive infiltrate of tumor infiltrating lymphocytes. (i) Membranous expression of PD-L1 was seen in 54\% of thymic carcinomas. (j) A spindle cell thymoma positive for PD-L1. (k) Among the conventional thymomas, 76\% were PD-L1 positive. (l) A case of atypical thymoma showing strong reactivity of PD-L1.

conventional thymomas (WHO B1 and B2) (76\%, Figure 1g), and 12/24 atypical thymomas (WHO B3) (50\%, Figure 1h). PD-L1 was detected in epithelial tumor cells. Positive PD-L1 cases included 14/26 thymic carcinomas (54\%, Figure 1i), 14/25 spindle cell thymomas ( $56 \%$, Figure 1j), 17/25 conventional thymomas (68\%, Figure $1 \mathrm{k})$, and $16 / 24$ atypical thymomas ( $67 \%$, Figure 11$)$. A total of $52 \%$ of thymic 
epithelial neoplasms were positive for PD-1 and 61\% for PD-L1. A total of $82 \%$ of cases showed expression of PD-1 or PD-L1 (thymic carcinoma: 16/26, 62\%; spindle cell thymoma: $21 / 25,84 \%$; conventional thymoma: $25 / 25,100 \%$; atypical thymoma: $20 / 24,83 \%)$.

\section{Clinical Follow-Up}

The median postoperative follow-up term was 80 months (range, 1-204 months). Among the 100 patients, 61 were alive and 36 had died. The deceased patients included 11 with thymic carcinoma, 2 of whom had died of causes other than thymic carcinoma and 25 thymoma patients, 11 of whom had died of unrelated causes.

\section{Statistical Analyses}

$P D-1$ and $P D-L 1$ expression and thymic carcinoma. PD-1 positive cases were associated with higher stage in thymic carcinoma $(P=0.01)$ but not with thymic carcinoma subtype (squamous cell carcinoma vs high-grade carcinoma, not otherwise specified vs basaloid carcinoma) $(P=0.86)$, lymph node status $(P=0.07)$, or neoadjuvant therapy $(P=0.51$, Table 2). Compared to thymoma, patients with thymic carcinoma were more likely to be PD-1 negative $(P=0.0014$, Table 3$)$. There was no evidence of a difference between PD-L1 positive and negative patients for any of these variables: tumor stage $(P=0.13)$, thymic carcinoma subtype $(P=0.08)$, lymph node status $(P=1)$, or neoadjuvant therapy $(P=0.08)$. There was no evidence of an association between tumor type (thymic carcinoma vs spindle cell thymoma vs conventional thymoma vs atypical thymoma) and PD-L1 status $(P=0.6572)$. Furthermore, there was no statistically significant difference in overall survival between PD-1 $(P=0.2137)$ and PD-L1 $(P=0.4628)$ positive and negative patients, respectively (Figures 2 and 3 ).
PD-1 and PD-L1 expression and thymoma. PD-L1 positive cases were associated with neoadjuvant therapy in thymoma patients $(P=0.0065)$ but not with tumor stage $(P=0.18)$ or tumor subtype (spindle cell vs conventional vs atypical thymoma) $(P=0.67$, Table 4). There was no evidence of a difference between PD-1 positive and negative patients for any of these variables: tumor stage $(P=0.11)$, tumor subtype $(P=0.15)$, or neoadjuvant therapy $(P=0.065)$. There was no evidence of an association between tumor type (thymic carcinoma vs spindle cell thymoma vs conventional thymoma vs atypical thymoma) and PD-L1 status $(P=0.6572)$. In addition, there was no statistically significant difference in overall survival between PD-1 $(P=0.3942)$ and PD-L1 $(P=0.4180)$ positive and negative patients, respectively (Figures 4 and 5).

\section{Discussion}

Immunotherapies targeting the PD-1/PD-L1 axis are a promising new therapeutic strategy for many tumor types. ${ }^{7-11}$ PD-1 is an immune checkpoint expressed on the surface of $\mathrm{T}$ lymphocytes on activation. ${ }^{15}$ It is quickly upregulated on $\mathrm{T}$ cells after exposure to certain antigens, waning upon antigen clearance. ${ }^{16}$ Under normal conditions, PD-1 signaling in T cells regulates immune responses to prevent tissue damage and counteract autoimmunity. Its ligand, PD-L1, a transmembrane glycoprotein, is constitutively expressed on $\mathrm{T}$ and $\mathrm{B}$ cells, macrophages and dendritic cells and is upregulated in many human cancer cells. ${ }^{17}$ Binding of PD-1 to PD-L1 inhibits the proliferation of activated $\mathrm{T}$ cells resulting in prevention of anti-tumor adaptive responses and tumor evasion of the immune system. Inhibitors of PD-1 and PD-L1 in the form of monoclonal antibodies disrupt this pathway and have been shown to promote significant and durable responses in cancer patients. ${ }^{5}$ Although expression of PD-1 and PD-L1 as detected by immunohistochemistry has been

Table 2 Thymic carcinoma patient characteristics by PD-1 positivity

\begin{tabular}{|c|c|c|c|c|c|c|c|}
\hline Variable & Levels & ${ }^{n}$ neg & ${ }^{\%}$ neg & ${ }^{n}$ pos & ${ }^{\%}$ pos & ${ }^{n}$ all & ${ }^{\%}$ all \\
\hline \multirow[t]{3}{*}{ Stage } & 1 & 8 & 42.1 & 0 & 0 & 8 & 32 \\
\hline & 2 & 9 & 47.4 & 2 & 33.3 & 11 & 44 \\
\hline & 3 & 2 & 10.5 & 4 & 66.7 & 6 & 24 \\
\hline$P=0.01$ & All & 19 & 100 & 6 & 100 & 25 & 100 \\
\hline \multirow[t]{3}{*}{ Histologic type } & Basaloid & 3 & 15 & 0 & 0 & 3 & 11.5 \\
\hline & High grade & 4 & 20 & 1 & 16.7 & 5 & 19.2 \\
\hline & Squamous cell & 13 & 65 & 5 & 83.3 & 18 & 69.2 \\
\hline$P=0.86$ & All & 20 & 100 & 6 & 100 & 26 & 100 \\
\hline \multirow{2}{*}{ Lymph node status } & Negative & 17 & 89.5 & 3 & 50 & 20 & 80 \\
\hline & Positive & 2 & 10.5 & 3 & 50 & 5 & 20 \\
\hline$P=0.07$ & All & 19 & 100 & 6 & 100 & 25 & 100 \\
\hline \multirow[t]{3}{*}{ Neoadjuvant chemotherapy } & Adj. & 1 & 5.3 & 0 & 0 & 1 & 4 \\
\hline & No & 5 & 26.3 & 3 & 50 & 8 & 32 \\
\hline & Yes & 13 & 68.4 & 3 & 50 & 16 & 64 \\
\hline$P=0.51$ & All & 19 & 100 & 6 & 100 & 25 & 100 \\
\hline
\end{tabular}


Table 3 Association between histology and PD-1

\begin{tabular}{lcc}
\hline Histology & Negative & Positive \\
\hline Thymic carcinoma & 20 & 6 \\
Thymoma/atypical & 12 & 12 \\
Thymoma/spindle cell & 10 & 15 \\
Thymoma/conventional & 6 & 19 \\
Total & 48 & 52 \\
\hline
\end{tabular}

The association between histology and PD-1 status (negative/positive) is highly significant $(P=0.0014)$. Patients with thymic carcinoma are more likely to be PD-1 negative.

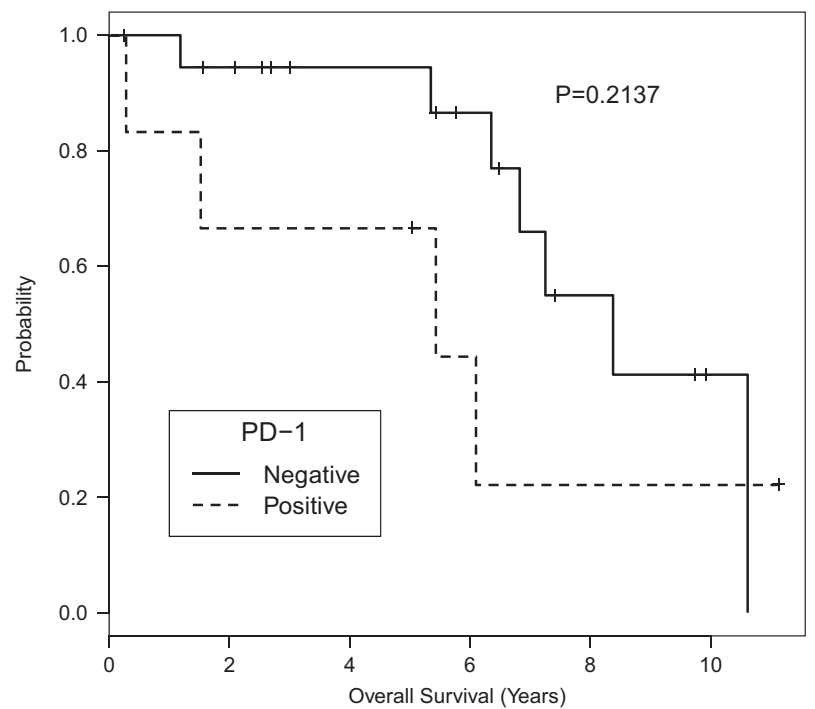

Figure 2 Kaplan-Meier overall survival curve for PD-1 status in thymic carcinoma.

associated with positive responses to anti-PD-1/PDL1 treatment, ${ }^{5,18}$ its prognostic value in different tumor types is still undetermined and limited data for thymic epithelial neoplasms have shown conflicting results. ${ }^{19-22}$

Four prior studies have addressed the immunohistochemical expression of PD-1 and/or PD-L1 in the context of thymic epithelial tumors. In 2015, Padda et $a 1^{19}$ evaluated the expression of PD-L1 in 69 cases using a tissue microarray; assessment of PD-L1 reactivity was based on an intensity score. The authors found that more intense PD-L1 staining was associated with higher grade WHO histology $(\mathrm{B} 3>\mathrm{B} 1=\mathrm{B} 2=\mathrm{C}>\mathrm{AB}>\mathrm{A})$, higher stage and worse clinical outcome. In the same year, a study from Japan $^{20}$ investigated the expression of PD-L1 in 101 thymomas and 38 thymic carcinomas arranged in a tissue microarray; a semiquantitative method was used for scoring the expression pattern. In this study, $70 \%$ of thymic carcinomas and $23 \%$ of thymoma samples showed positive staining for PD-L1. Furthermore, the authors concluded that although thymic carcinoma was significantly associated with PD-L1 expression, other variables including age at diagnosis, sex, tumor stage, tumor size, neoadjuvant therapy, and overall survival did not show any

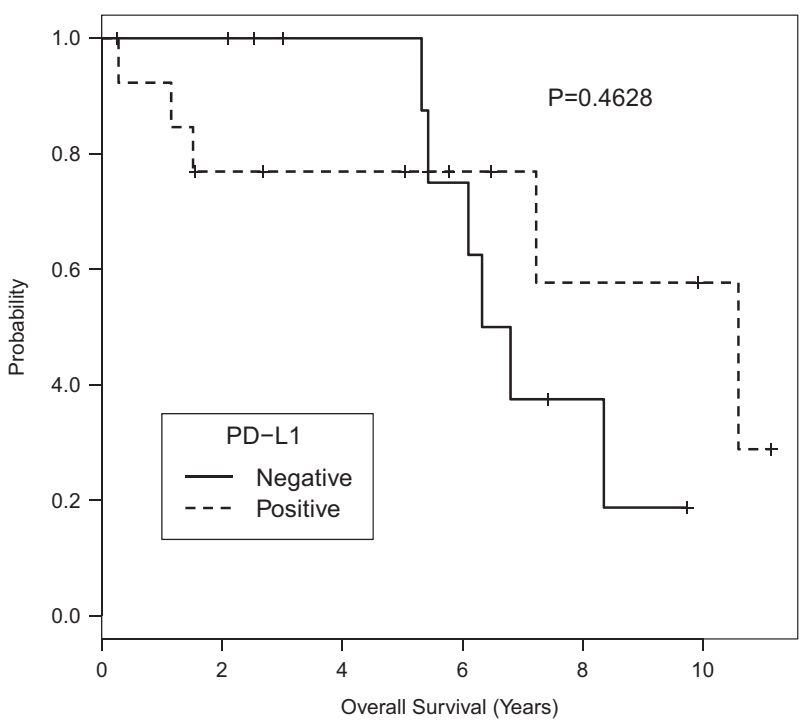

Figure 3 Kaplan-Meier overall survival curve for PD-L1 status in thymic carcinoma.

Table 4 Thymoma patient characteristics by PD-L1 positivity

\begin{tabular}{lrrrrrr}
\hline Variable levels & ${ }^{n}$ neg & ${ }^{\%}$ neg & ${ }^{n}$ pos & ${ }^{\%}$ pos & ${ }^{n}$ all & ${ }^{\%}$ all \\
\hline Moran stage & & & & & & \\
$\quad 0$ & 4 & 15.4 & 4 & 8.7 & 8 & 11.1 \\
1 & 16 & 61.5 & 17 & 37 & 33 & 45.8 \\
2a & 1 & 3.8 & 9 & 19.6 & 10 & 13.9 \\
2b & 2 & 7.7 & 3 & 6.5 & 5 & 6.9 \\
2C & 0 & 0 & 1 & 2.2 & 1 & 1.4 \\
3a & 3 & 11.5 & 11 & 23.9 & 14 & 19.4 \\
3b & 0 & 0 & 1 & 2.2 & 1 & 1.4 \\
P.18; all & 26 & 100 & 46 & 100 & 72 & 100 \\
& & & & & & \\
Histologic type & & & & & & \\
$\quad$ Atypical & 8 & 29.6 & 16 & 34 & 24 & 32.4 \\
Spindle & 11 & 40.7 & 14 & 29.8 & 25 & 33.8 \\
Thymoma & 8 & 29.6 & 17 & 36.2 & 25 & 33.8 \\
P=0.67; all & 27 & 100 & 47 & 100 & 74 & 100 \\
& & & & & &
\end{tabular}

Neoadjuvant

chemotherapy

\begin{tabular}{lrccccc} 
Adj. & 1 & 4 & 0 & 0 & 1 & 1.4 \\
No & 15 & 60 & 14 & 30.4 & 29 & 40.9 \\
Yes & 9 & 36 & 32 & 69.6 & 41 & 57.8 \\
$P=0.0065 ;$ all & 25 & 100 & 46 & 100 & 71 & 100 \\
\hline
\end{tabular}

association with PD-L1 status. In the two most recent reports, Yokoyama et $a l^{21}$ separately investigated thymomas $(n=82)$ and thymic carcinomas $(n=25) .^{22}$ In their analysis of thymomas, ${ }^{21} 54 \%$ of cases revealed high expression of PD-L1 and PD-L1 high status was associated with high tumor stage, highgrade WHO histology, worse disease-free survival, and increased rate of recurrence but not with overall survival. Contrary to that, they found that high PD-L1 expression was associated with a higher disease-free and overall survival in thymic carcinomas, whereas an increased number of $\mathrm{PD}-1^{+}$tumor infiltrating lymphocytes correlated with poor prognosis in this cohort. $^{22}$ 


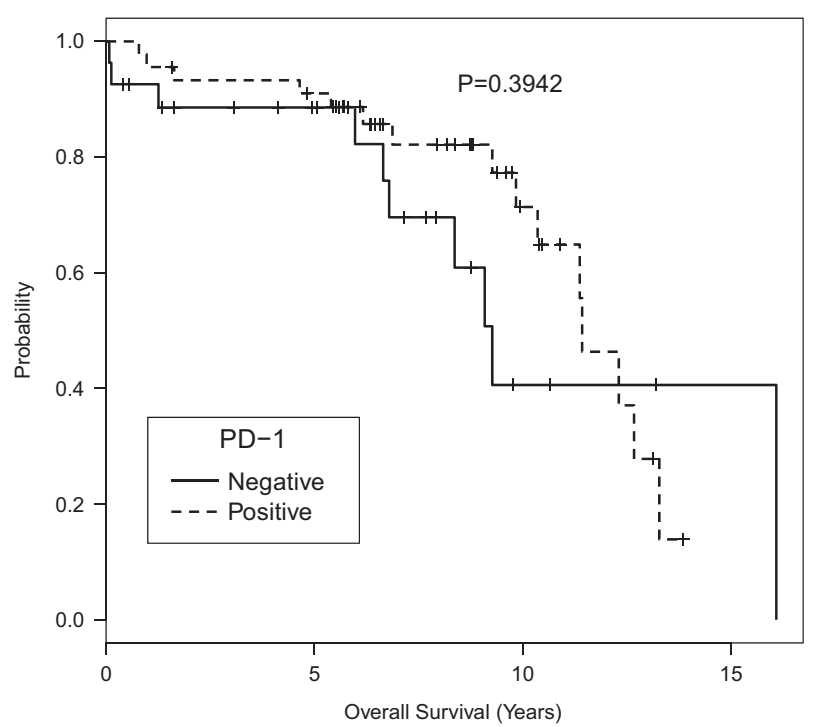

Figure 4 Kaplan-Meier overall survival curve for PD-1 status in thymoma.

Our study confirmed high expression of PD-1 $(52 \%)$ and PD-L1 $(61 \%)$ in thymic epithelial neoplasms (with positive rates for PD-1 of $62 \%$ in thymoma and $23 \%$ in thymic carcinoma, respectively, and positive rates for PD-L1 of $64 \%$ in thymoma and $54 \%$ in thymic carcinoma, respectively), supporting the results of the previous studies. On the other hand, our results could not confirm that PD-L1 expression is correlated with tumor histology as there was no statistical difference in expression when comparing spindle cell thymoma, conventional thymoma, atypical thymoma and thymic carcinoma. Likewise, we could not support the results by Padda et $a l^{19}$ and Yokoyama et $a l^{21}$ that PD-L1 expression is significantly associated with tumor stage. In terms of survival, worse outcome for PD-L1 positive cases has been documented in the study by Padda et $a l^{19}$ who noticed shorter overall survival in tumors with high expression of PD-L1, whereas Yokoyama et $a l^{21}$ described worse diseasefree survival and increased recurrence rates but no change in overall survival among their thymoma cohort. Overall survival was also unchanged in the series by Katsuya et $a{ }^{20}{ }^{20}$ a finding that seems to be supported by our study although follow up was generally limited. The only variable that was associated with PD-L1 expression in our cases was neoadjuvant treatment in cases of thymoma but not thymic carcinoma; in this context, PD-L1 positive cases were more frequently found in patients with thymoma who had received neoadjuvant chemotherapy. Recently, neoadjuvant chemoradiation therapy was found to be associated with an increase in the proportion of PD-L1+ tumor cells in patients with rectal adenocarcinoma ${ }^{23}$ leading the authors to propose the use of combination therapy consisting of radiochemotherapy and PD-1/PD-L1

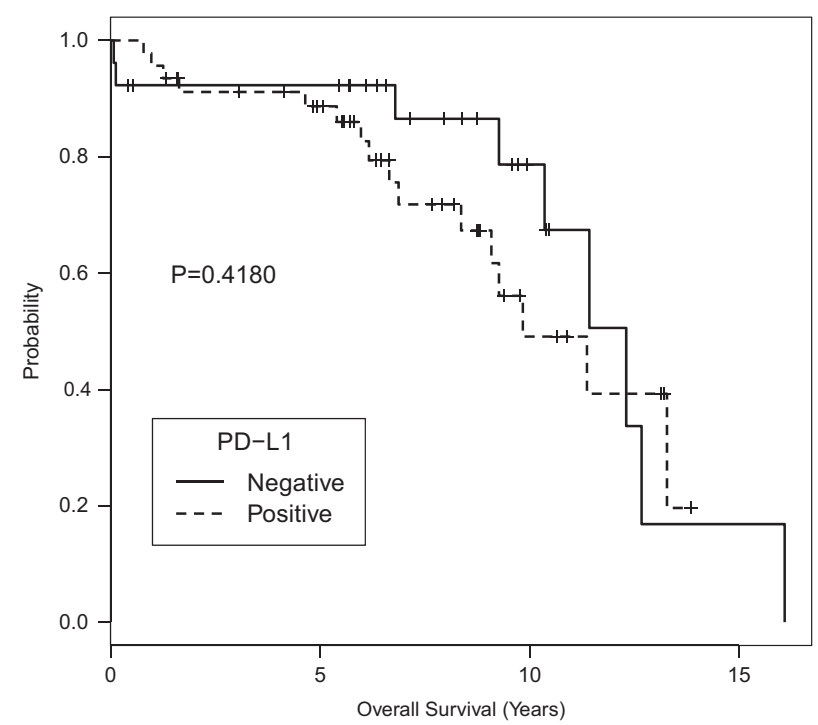

Figure 5 Kaplan-Meier overall survival curve for PD-L1 status in thymoma.

blockade in the treatment of these tumors. Whether such combination treatment would also be advised in thymic epithelial neoplasms requires further study as our results are in conflict with those of Katsuya et $a l^{20}$ who found no influence of neoadjuvant treatment on PD-L1 expression in these tumors. Furthermore, in our series, expression of PD-L1 was not associated with any of the examined variables in thymic carcinoma including tumor stage, carcinoma subtype, lymph node status, neoadjuvant therapy, and overall survival in agreement with the results by Katsuya et $a l^{19}$ for this tumor group. In contrast, although Yokoyama et $a l^{22}$ in their study on thymic carcinomas also found no correlation of PD-L1 status with patient age, sex, tumor stage, tumor histology, or surgical curability, they did observe an increased disease-free and overall survival in cases with high PD-L1 expression.

PD-1 has only been incompletely studied in thymic epithelial neoplasms. In fact, expression of PD-1 was only addressed in the study by Katsuya et $a l^{20}$ who exclusively applied this antibody to their group of thymic carcinomas but not thymomas and in the study by Yokoyama et $a l^{22}$ in their study on thymic carcinomas. Katsuya et al found that PD-1 positivity was restricted to tumor infiltrating lymphocytes of $62 \%$ of their cases but an association between expression of PD-1 and PD-L1 as had been suggested by prior studies in different cancer types ${ }^{24,25}$ could not be confirmed. In the study by Yokoyama et $a{ }^{22}$ an increased number of $\mathrm{PD}-1^{+}$ tumor infiltrating lymphocytes was associated with poor prognosis in thymic carcinomas. The results of our own study showed a much lower expression of PD-1 in the tumor infiltrating lymphocytes of thymic carcinomas $(23 \%)$ compared to a significantly higher rate in thymomas $(62 \%, P=0.0014)$. In the past, an 
increase in $\mathrm{PD}-1^{+}$tumor infiltrating lymphocytes has been suggested to reflect a favorable prognostic factor for various cancer types, ${ }^{26-30}$ however in our series, no difference in survival between PD-1positive and negative cases was observed. Another positive association in our study was between PD-1 positive cases and higher tumor stage in cases of thymic carcinoma. Similar results have recently been observed in melanoma patients and were interpreted to represent stronger functional impairment of PD-1 ${ }^{+} \mathrm{T}$ cells in advanced stages of the disease. Similar to our study, an effect on overall survival could not be observed in that study. ${ }^{31}$ Furthermore, expression of PD-1 was not associated with any parameters in thymoma patients nor with tumor subtype, lymph node status, or neoadjuvant therapy in cases of thymic carcinoma.

Overall, our results confirm the observation that PD-1 and PD-L1 are highly expressed in thymic epithelial neoplasms suggesting that anti-PD-1/PDL1 drugs may be a promising alternative treatment for these tumors. On the other hand, we could not support the prior opinion that expression of PD-L1 is associated with higher grade tumor histology, higher tumor stage, or is related to prognosis. Thus, controversy still remains about the use of these biomarkers for prognostic purposes in thymic epithelial neoplasms. Such controversy is not unique to these tumors but has been noted for a variety of other cancers in this context. For instance, the correlation of high PD-L1 expression with clinical progression and shortened overall survival has been described for melanoma, renal cell carcinoma, and non-small cell lung cancer, ${ }^{10,32,33}$ but improved prognosis has been noted for PD-L1-positive colorectal carcinomas and ovarian cancers. ${ }^{30,34,35}$ Some of these discrepancies may be explained by certain limitations when comparing different studies. These primarily appear to lie in the study design and include the type of tissue studied (fresh vs formalinfixed paraffin-embedded), the amount of tissue available for examination (tissue microarray $v s$ whole-tissue sections), the use of antibody clones with different specificity, highly variable definition of PD-1/PD-L1 positivity and the method of scoring the results. Another complicating factor in the analysis of thymoma in this context is the common heterogeneous composition of tumors and inclusion of mixed histologic types in previous study cohorts ${ }^{19}$ raising concern about the validity of results especially when correlating PD-1/PD-L1 expression status with histologic subtypes. Unless more uniform study designs are applied, direct comparison of studies will remain difficult and the results need to be interpreted with a certain degree of caution.

In conclusion, our results support that PD-1 and PD-L1 are highly expressed in thymic epithelial neoplasms raising the possibility of the use of anti-PD-1/PD-L1 therapy in the treatment of these tumors. In contrast, neither expression of PD-1 nor PD-L1 seemed to be associated with patient outcome in our group, adding uncertainty to the role of these markers for prognostic purposes. The fact that PD-L1 expression was associated with neoadjuvant treatment in thymoma raises the possibility of use of combination therapy in such tumors, however further studies to this effect are required.

\section{Acknowledgments}

The study was funded by an institutional philanthropic research grant (600663-80-112487-19).

\section{Disclosure/conflict of interest}

The authors declare no conflict of interest.

\section{References}

1 Engels EA, Pfeiffer RM. Malignant thymoma in the United States: demographic patterns in incidence and associations with subsequent malignancies. Int J Cancer 2003;105:546-551.

2 Engels EA. Epidemiology of thymoma and associated malignancies. J Thorac Oncol 2010;5:S260-S265.

3 Weissferdt A, Wistuba II, Moran CA. Molecular aspects of thymic carcinoma. Lung Cancer 2012;78:127-132.

4 Pardoll DM. The blockade of immune checkpoints in cancer immunotherapy. Nat Rev Cancer 2012;12: 252-264.

5 Topalian SL, Hodi FS, Brahmer JR, et al. Safety, activity, and immune correlates of anti-PD-1 antibody in cancer. N Engl J Med 2012;366:2443-2454.

6 Flemming A. Cancer: PD1 makes waves in anticancer immunotherapy. Nat Rev Drug Discov 2012;11:601.

7 Chen BJ, Chapuy B, Ouyang J, et al. PD-L1 expression is characteristic of a subset of aggressive B-cell lymphomas and virus-associated malignancies. Clin Cancer Res 2013;19:3462-3473.

8 Yang CY, Lin MW, Chang YL, et al. Programmed cell death-ligand 1 expression in surgically resected stage I pulmonary adenocarcinoma and its correlation with driver mutations and clinical outcomes. Eur J Cancer 2014;50:1361-1369.

9 Berghoff AS, Ricken G, Widhalm G, et al. PD1 (CD279) and PD-L1 (CD274, B7H1) expression in primary central nervous system lymphomas (PCNSL). Clin Neuropathol 2014;33:42-49.

10 Zhang Y, Wang L, Li Y, et al. Protein expression of programmed death 1 ligand 1 and ligand 2 independently predict poor prognosis in surgically resected lung adenocarcinoma. Onco Targets Ther 2014;7: 567-573.

11 Merelli B, Massi D, Cattaneo L, et al. Targeting the PD1/PD-L1 axis in melanoma: biological rationale, clinical challenges and opportunities. Crit Rev Oncol Hematol 2014;89:140-165.

12 Suster S, Moran CA. Thymoma, atypical thymoma, and thymic carcinoma. A novel conceptual approach to the classification of thymic epithelial neoplasms. Am J Clin Pathol 1999;111:826-833.

13 Moran CA, Walsh G, Suster S, et al. Thymomas II: a clinicopathologic correlation of 250 cases with a 
proposed staging system with emphasis on pathologic assessment. Am J Clin Pathol 2012;137:451-461.

14 Weissferdt A, Moran CA. Thymic carcinoma, part 2: a clinicopathologic correlation of 33 cases with a proposed staging system. Am J Clin Pathol 2012;138:115-121.

15 Keir ME, Butte MJ, Freeman GJ, et al. PD-1 and its ligands in tolerance and immunity. Annu Rev Immunol 2008;26:677-704.

16 Kinter AL, Godbout EJ, McNally JP, et al. The common gamma-chain cytokines IL-2, IL-7, IL-15, and IL-21 induce the expression of programmed death-1 and its ligands. J Immunol 2008;181:6738-6746.

17 Dong H, Strome SE, Salomao DR, et al. Tumor-associated B7-H1 promotes T-cell apoptosis: a potential mechanism of immune evasion. Nat Med 2002;8:793-800.

18 Brahmer JR, Drake CG, Wollner I, et al. Phase I study of single-agent anti-programmed death-1 (MDX-1106) in refractory solid tumors: safety, clinical activity, pharmacodynamics, and immunologic correlates. Clin Oncol 2010;28:3167-3175.

19 Padda SK, Riess JW, Schwartz EJ, et al. Diffuse high intensity PD-L1 staining in thymic epithelial tumors. J Thorac Oncol 2015;10:500-508.

20 Katsuya Y, Fujita Y, Horinouchi H, et al. Immunohistochemical status of PD-L1 in thymoma and thymic carcinoma. Lung Cancer 2015;88:154-159.

21 Yokoyama S, Miyoshi H, Nishi T, et al. Clinicopathologic and prognostic implications of programmed death ligand 1 expression in thymoma. Ann Thorac Surg 2016;101:1361-1369.

22 Yokoyama S, Miyoshi H, Nakashima K, et al. Prognostic value of programmed death ligand 1 and programmed death 1 expression in thymic carcinoma. Clin Cancer Res 2016;22:4727-4734.

23 Hecht M, Büttner-Herold M, Erlenbach-Wünsch K, et al. PD-L1 is upregulated by radiochemotherapy in rectal adenocarcinoma patients and associated with a favourable prognosis. Eur J Cancer 2016;65:52-60.

24 Taube JM, Klein A, Brahmer JR, et al. Association of PD-1, PD-1 ligands, and other features of the tumor immune microenvironment with response to anti-PD-1 therapy. Clin Cancer Res 2014;20:5064-5074.

25 Konishi J, Yamazaki K, Azuma M, et al. B7-H1 expression on non-small cell lung cancer cells and its relationship with tumor-infiltrating lymphocytes and their PD-1 expression. Clin Cancer Res 2004;10: 5094-5100.

26 Liu H, Zhang $\mathrm{T}$, Ye J, et al. Tumor-infiltrating lymphocytes predict response to chemotherapy in patients with advance non-small cell lung cancer. Cancer Immunol Immunother 2012;61:1849-1856.

27 Hamanishi J, Mandai M, Iwasaki M, et al. Programmed cell death 1 ligand 1 and tumor-infiltrating CD8+ T lymphocytes are prognostic factors of human ovarian cancer. Proc Natl Acad Sci USA 2007;104: 3360-3365.

28 Al-Shibli KI, Donnem T, Al-Saad S, et al. Prognostic effect of epithelial and stromal lymphocyte infiltration in non-small cell lung cancer. Clin Cancer Res 2008;14: 5220-5227.

29 Badoual C, Hans S, Merillon N, et al. PD-1-expressing tumor-infiltrating $\mathrm{T}$ cells are a favorable prognostic biomarker in HPV-associated head and neck cancer. Cancer Res 2013;73:128-138.

$30 \mathrm{Li} \mathrm{Y}$, Liang L, Dai W, et al. Prognostic impact of programed cell death-1 (PD-1) and PD-ligand 1 (PD-L1) expression in cancer cells and tumor infiltrating lymphocytes in colorectal cancer. Mol Cancer 2016;15: 55.

31 Krönig H, Julia Falchner K, Odendahl M, et al. PD-1 expression on Melan-A-reactive $\mathrm{T}$ cells increases during progression to metastatic disease. Int J Cancer 2012;130:2327-2336.

32 Oba J, Nakahara T, Abe $\mathrm{T}$, et al. Expression of programmed death receptor ligand 1 in melanoma may indicate tumor progression and poor patient survival. J Am Acad Dermatol 2014;70:954-956.

33 Thompson RH, Dong $\mathrm{H}$, Kwon ED. Implications of B7-H1 expression in clear cell carcinoma of the kidney for prognostication and therapy. Clin Cancer Res 2007;13:709s-715s

34 Droeser RA, Hirt C, Viehl CT, et al. Clinical impact of programmed cell death ligand 1 expression in colorectal cancer. Eur J Cancer 2013;49:2233-2242.

35 Webb JR, Milne K, Kroeger DR, et al. PD-L1 expression is associated with tumor-infiltrating $\mathrm{T}$ cells and favorable prognosis in high-grade serous ovarian cancer. Gynecol Oncol 2016;141:293-302. 